\title{
Stability in Matching with Couples having Responsive Preferences
}

Citation for published version (APA):

Khare, S., Roy, S., \& Storcken, T. (2021). Stability in Matching with Couples having Responsive

Preferences. Social Science Research Network (SSRN). SSRN Working Paper Series No. 3288199 https://doi.org/10.2139/ssrn.3288199

Document status and date:

Published: 01/02/2021

DOI:

10.2139/ssrn.3288199

\section{Please check the document version of this publication:}

- A submitted manuscript is the version of the article upon submission and before peer-review. There can be important differences between the submitted version and the official published version of record.

People interested in the research are advised to contact the author for the final version of the publication, or visit the DOI to the publisher's website.

- The final author version and the galley proof are versions of the publication after peer review.

- The final published version features the final layout of the paper including the volume, issue and page numbers.

Link to publication

\footnotetext{
General rights rights.

- You may freely distribute the URL identifying the publication in the public portal. please follow below link for the End User Agreement:

www.umlib.nl/taverne-license

Take down policy

If you believe that this document breaches copyright please contact us at:

repository@maastrichtuniversity.nl

providing details and we will investigate your claim.
}

Copyright and moral rights for the publications made accessible in the public portal are retained by the authors and/or other copyright owners and it is a condition of accessing publications that users recognise and abide by the legal requirements associated with these

- Users may download and print one copy of any publication from the public portal for the purpose of private study or research.

- You may not further distribute the material or use it for any profit-making activity or commercial gain

If the publication is distributed under the terms of Article $25 \mathrm{fa}$ of the Dutch Copyright Act, indicated by the "Taverne" license above, 


\title{
Stability in Matching with Couples having Responsive Preferences*
}

\author{
Shashwat Khare ${ }^{1}$, Souvik Roy ${ }^{\dagger 11,2}$, and Ton Storcken ${ }^{1}$ \\ ${ }^{1}$ Quantitative Economics, Maastricht University \\ ${ }^{2}$ Economics Research Unit, Indian Statistical Institute
}

February 27, 2021

\begin{abstract}
This paper studies matching markets where institutions are matched with possibly more than one individual. The matching market contains some couples who view the pair of jobs as complements. First, we show by means of an example that a stable matching may fail to exist even when both couples and institutions have responsive preferences. Next, we provide conditions on couples' preferences that are necessary and sufficient to ensure a stable matching for every preference profile where institutions may have any responsive preference. Finally, we do the same with respect to institutions' preferences, that is, we provide conditions on institutions' preferences that are necessary and sufficient to ensure a stable matching for every preference profile where couples may have any responsive preference.
\end{abstract}

*This paper would not have been possible without the valuable inputs and suggestions by Dr. Hans Peters.

${ }^{\dagger}$ Corresponding author: souvik.2004@gmail.com

†This project has received funding from the European Union's Horizon 2020 research and innovation program under the Marie-Sklodowska-Curie grant agreement N0 660341. 
KEYWORDS. two-sided matching, stability, responsiveness

JEL Classification Codes. C78, D47

\section{Introduction}

\subsection{Background of the problem}

A huge literature has been developed on various market designs for finding an "optimal" matching procedure in a labour market. In many centralized labour markets, stability is an important condition for optimality. A matching is stable when there do not exist institution-individual pairs that are not matched to each other, but when being matched are better off compared to their present allocation. Such pairs are also known as blocking pairs. For labour markets consisting of institutions (referred to here as hospitals) and individuals (here, called doctors), stable matchings do exist under appropriate domain restrictions.

Roth[12] was the first to point out that the presence of couples in labour markets may exclude the existence of a stable matching. This can happen because couples may view pairs of jobs as complements and therewith the assumption on the independence of the individual choices might not apply.

However, Kelso and Crawford[8], Roth[13], Alkan and Gale[2] and Hatfield and Milgrom[7] show that a sufficient amount of substitutability can guarantee the existence of a stable matching. These papers assume substitutability of preferences of the hospitals over sets of doctors. Later, Klaus and Klijn[9] assume responsiveness of couples' preferences over ordered pairs of hospitals and show that the existence of a stable matching is guaranteed in such settings. Responsiveness means that unilateral improvements according to the preference of one couple member are beneficial for the couple. 


\subsection{Our motivation}

As mentioned, we assume that the institutions are hospitals and the individuals are doctors, where the subset of doctors includes couples. We assume that couples' preferences are responsive over the pair of hospitals and hospitals' preferences are responsive over the set of doctors.

It is interesting to note that given the individual preferences of the couple members, more than one (joint) couple preference satisfies responsiveness. For example, suppose we have a couple $c=\{f, m\}$ and two hospitals $h_{1}$ and $h_{2}$ such that $f$ prefers $h_{1}$ to $h_{2}$ but $m$ prefers $h_{2}$ to $h_{1}$. Suppose further that $(x, y)$ denotes an allocation of this couple where $f$ is matched with $x$ and $m$ is matched with $y$. Then we know by responsiveness that $\left(h_{1}, h_{2}\right)$ is preferred to both $\left(h_{1}, h_{1}\right)$ and $\left(h_{2}, h_{2}\right)$ whereas, $\left(h_{1}, h_{1}\right)$ and $\left(h_{2}, h_{2}\right)$ are both preferred to $\left(h_{2}, h_{1}\right)$. However, responsiveness does not give us an ordering between the pairs $\left(h_{1}, h_{1}\right)$ and $\left(h_{2}, h_{2}\right)$. Thus there are different complete responsive couple preference extensions. Similarly, given a preference of a hospital over doctors, we can have more than one responsive preference over subsets of doctors.

Allowing for complete responsive preferences for both couples of doctors and hospitals is not innocuous, as in such situations stable matchings may fail to exist. On the other hand, allowing that the pairs $\left(h_{1}, h_{1}\right)$ and $\left(h_{2}, h_{2}\right)$ are comparable in the above mentioned example of couple preference seems to be natural. Similarly, it seems natural that hospitals admitting teams of doctors compare, for instance, two pairs of doctors, where in one pair, there is one $\mathrm{A}$ ranked doctor and one $\mathrm{C}$ ranked doctor, and in the other pair there are two B ranked doctors. As we will see, allowing for such comparisons does affect the stability of matchings. However, it is worthwhile to mention that such comparisons might not always be possible even when the preferences of hospitals satisfy responsiveness. For example, by a standard replication of hospitals up to their capacities to create a one-to-one matching, these pairs of doctors are actually considered incomparable. This is because, for the hospital the $\mathrm{B}$ ranked doctor is better than the $\mathrm{C}$ ranked doctor but is 
worse than the A ranked doctor. Thus, replication implies that these two sets of doctors are incomparable. The possibility of allowing for all such above mentioned comparisons is the main reason why results presented here differ from Klaus and Klijn[9], who found stable matchings in every case of couples' responsive preferences.

\subsection{Our contribution}

In this paper, we formalize our idea of complete responsive preferences of hospitals and couples and study its consequences for the existence of a stable matching.

First, we show by means of an example that stable matchings may not exist at arbitrary responsive preference profiles. Then, we provide a condition on couples' preferences that is necessary and sufficient for guaranteeing the existence of a stable matching for every responsive extension of hospitals' preferences over sets of doctors. We call this condition extreme-altruism. We explain this condition by means of the following example. Suppose that there are $k$ hospitals $\left\{h_{1}, \ldots, h_{k}\right\}$ and a couple. Suppose further that $h_{i}$ is preferred to $h_{j}$ for all $1 \leq i<j \leq k$ by both members of the couple. Then, extreme-altruism says that, for any $1 \leq i<j \leq k$, the couple prefers the allocation where one member is matched with $h_{i}$ and the other is matched with $h_{k}$ compared to the allocation where both of them are matched with $h_{j}$.

Next, we provide conditions on hospitals' preferences that are necessary and sufficient for guaranteeing the existence of a stable matching for every responsive extension of couples' preferences. We call this condition aversion to couple diversity. We explain this condition by means of the following example. Consider a hospital $h$ with preference $P_{h}$ over individual doctors. Let $c=\{f, m\}$ be a couple and let $d, d^{\prime}$ be two other doctors (possibly another couple). Suppose that $f P_{h} d P_{h} d^{\prime} P_{h} m$. As we have discussed earlier, responsiveness does not put any restriction on $h$ 's preference over the relative ordering of the sets of doctors $\{f, m\}$ and $\left\{d, d^{\prime}\right\}$. A hospital is averse of diverse couples if in such situations it prefers $\left\{d, d^{\prime}\right\}$ to $\{f, m\}$. Loosely put, 
this property asserts that a hospital does not like to employ couples whose members are relatively more dissimilar (with respect to its preference over individual doctors).

Another important contribution of our paper is that, not only we characterize profiles where stable matchings exist, but we also provide algorithms that produce a stable matching whenever that exists.

Thus, we feel that our paper provides a more complete picture on the issue of the existence of stable matchings when hospitals and couples have responsive preferences, and consequently complements the work of Klaus and Klijn[9].

\subsection{Organization of the paper}

In the next section, we lay out the framework of our model and formally provide all the necessary definitions. We also state and describe an algorithm for matching doctors with hospitals, which we use throughout this paper. In Section 3, we provide an example showing that the existence of a stable matching is not guaranteed at arbitrary responsive preferences profiles. In Section 4, we present conditions on couples' preferences that are necessary and sufficient for guaranteeing the existence of a stable matching for arbitrary responsive extensions hospitals' preferences, and in Section 5, we present conditions on hospitals' preferences that are necessary and sufficient for guaranteeing the existence of a stable matching for arbitrary responsive extensions of couples' preferences. We conclude with Section 6 where we explain the difference between our paper and Klaus and Klijn[9] by means of a formal example.

\section{The framework}

We consider many-to-one matchings between doctors and hospitals. We denote by $H$ a finite set of hospitals. Each hospital $h \in H$ has a finite capacity, denoted by $\kappa_{h}$. 
We denote by $D$ a finite set of doctors. We assume that $D=M \cup F \cup S$ where $F, M, S$ are pairwise disjoint sets with $|M|=|F|$. Here, the doctors in $F$ and $M$ together form fixed couples. Also, the doctors in $S$ are those who are not part of any couple. We denote the set of couples by $C=$ $\left\{\left\{f_{1}, m_{1}\right\},\left\{f_{2}, m_{2}\right\}, \ldots\right\}$ and a generic couple by $c=\{f, m\}$. We denote by $\lambda \notin H$, a dummy hospital which we use to represent a doctor being unemployed.

Throughout this paper, we assume $|H| \geq 2,|D| \geq 4,|C| \geq 1$, and $\kappa_{h} \geq 2$ for all $h \in H$ and $\kappa_{\lambda}=|D|$.

For notational convenience, we do not always use braces for denoting singleton hospitals, doctors or couples.

\subsection{Matching}

Definition 1 A matching $\mu$ is a correspondence from $H \cup\{\lambda\}$ to $D$ such that for all $h \in H,|\mu(h)| \leq \kappa_{h}$. Moreover $\mu\left(h_{1}\right) \cap \mu\left(h_{2}\right)=\emptyset$ for any $h_{1}, h_{2} \in H$ with $h_{1} \neq h_{2}$.

For ease of notation, whenever $d \in \mu(h)$ for some $d \in D$ and $h \in H$, we write $\mu(d)=h$. We say that a doctor is matched with $\lambda$ to mean that the doctor is unemployed. More formally, if $d \notin \mu(h)$ for all $h \in H$, then $\mu(d)=\lambda$. For a couple $c=\{f, m\} \in C$ and for hospitals $h, h^{\prime} \in H \cup\{\lambda\}$, we write $\mu(c)=\left(h, h^{\prime}\right)$ to mean $\mu(f)=h$ and $\mu(m)=h^{\prime}$. Further, for a hospital $h \in H$ and a matching $\mu$, we say $h$ has $\kappa_{h}-|\mu(h)|$ vacant positions at $\mu$.

\section{$2.2 \quad$ Preferences}

In this section, we introduce the notion of preferences of doctors and hospitals, and present some restrictions on them.

For a set $X$, we denote by $\mathbb{L}(X)$ the set of linear orders on $X$, i.e., complete, reflexive, transitive, and antisymmetric binary relations over $X$. An element of $\mathbb{L}(X)$ is called a preference (over $X$ ). For any $i \in H \cup D \cup C$, $R_{i}$ denotes a preference of $i$ and $P_{i}$ denotes its strict part. Since a preference 
is antisymmetric $x R_{i} y$ implies either $x=y$ or $x P_{i} y$. We say $x$ is weakly preferred to $y$ to mean $x R_{i} y$, and $x$ is (strictly) preferred to $y$ to mean $x P_{i} y$. For $P_{i} \in \mathbb{L}(X)$ and $k \leq|X|$, we define the $k$-th ranked element in $P_{i}$, denoted by $r_{k}\left(P_{i}\right)$, as follows: $r_{k}\left(P_{i}\right)=x \in X$ if $\left|\left\{y \in X: y R_{i} x\right\}\right|=k$.

\subsubsection{Preferences of hospitals}

For any hospital $h \in H$, let $D_{h}$ be the set of acceptable doctors. A preference of hospital $h$, denoted by $\bar{P}_{h}$, is a linear order over $D_{h}$. Thus, $\bar{P}_{h} \in \mathbb{L}\left(D_{h}\right)$. A hospital prefers to have any doctor from this set of acceptable doctors, over having a vacant spot. Similarly, a hospital prefers to have a vacant spot to having doctors which do not belong to the set of acceptable doctors. We assume that the dummy hospital $\lambda$ finds all doctors acceptable. Thus, $D_{\lambda}=D$. Also, $\lambda$ is indifferent between all doctors.

For any hospital $h \in H$, a preference $\bar{P}_{h}$ over individual doctors is extended to a preference $P_{h}$ over feasible subsets of acceptable doctors $\left\{D^{\prime} \subseteq\right.$ $\left.D_{h}:\left|D^{\prime}\right| \leq \kappa_{h}\right\}$.

Definition 2 We say $P_{h} \in \mathbb{L}\left(\left\{D^{\prime} \subseteq D_{h}:\left|D^{\prime}\right| \leq \kappa_{h}\right\}\right)$ is responsive if

(i) for all $D^{\prime} \subseteq D_{h}$ with $D^{\prime} \neq \emptyset$ and $\left|D^{\prime}\right| \leq \kappa_{h}, D^{\prime} P_{h} \emptyset$.

(ii) for all $d, d^{\prime} \in D_{h},\{d\} R_{h}\left\{d^{\prime}\right\}$ if and only if $d \bar{R}_{h} d^{\prime}$, and

(iii) for all $D^{\prime}, D^{\prime \prime} \subseteq D_{h}$ with $\left|D^{\prime}\right|<\kappa_{h},\left|D^{\prime \prime}\right|<\kappa_{h}$ and all $d \in D_{h} \backslash\left(D^{\prime} \cup D^{\prime \prime}\right)$, $\left(D^{\prime} \cup\{d\}\right) P_{h}\left(D^{\prime \prime} \cup\{d\}\right)$ if and only if $D^{\prime} P_{h} D^{\prime \prime}$.

Having define $P_{h}$ over all feasible subsets of acceptable doctors, we extend this preference over the set of all feasible subsets of doctors $\left\{D^{\prime} \subseteq D:\left|D^{\prime}\right| \leq\right.$ $\left.\kappa_{h}\right\}$.

Definition 3 For all $D^{\prime} \subseteq D$ such that $\left|D^{\prime}\right| \leq \kappa_{h}$ and $D^{\prime} \not \subset D_{h}$, we have $\emptyset P_{h} D^{\prime}$.

For notational convenience, for any hospital $h \in H$, any couple $c=$ $\{f, m\} \in C$ and any doctor $d \in D \backslash\{f, m\}, d P_{h} c$ means $d P_{h} f$ and $d P_{h} m$. 


\subsubsection{Preferences of doctors and couples}

A preference of a doctor $d \in D$, denoted by $P_{d}$, is an element of $\mathbb{L}(H \cup\{\lambda\})$. A preference of a couple $c=\{f, m\} \in C$, denoted by $P_{c}$, is an element of $\mathbb{L}\left((H \cup\{\lambda\})^{2}\right)$. We call a preference of a couple responsive if a unilateral improvement in the position of one member of the couple is beneficial for the couple.

Definition 4 Let $c=\{f, m\} \in C$ be a couple. Let $P_{f}$ be a preference of $f$ and $P_{m}$ be a preference of $m$. A preference $P_{c} \in \mathbb{L}\left((H \cup\{\lambda\})^{2}\right)$ of the couple c is called responsive (with respect to $P_{f}$ and $P_{m}$ ) if for all $h, h_{1}, h_{2} \in H \cup\{\lambda\}$, we have

(i) $\left(h_{1}, h\right) P_{c}\left(h_{2}, h\right)$ if and only if $h_{1} P_{f} h_{2}$, and

(ii) $\left(h, h_{1}\right) P_{c}\left(h, h_{2}\right)$ if and only if $h_{1} P_{m} h_{2}$.

For any $c=\{f, m\} \in C$, a responsive preference $P_{c}$ induces unique marginal preferences $P_{f}$ and $P_{m}$ for $f$ and $m$ respectively.

\subsubsection{Preference profiles and matching problems}

A preference profile is a collection of responsive preferences for all hospitals in $H$, all doctors in $D$ and all couples in $C$. Thus, a preference profile $P$ is a tuple of preferences $\left(\left\{P_{d}\right\}_{d \in D},\left\{P_{c}\right\}_{c \in C},\left\{P_{h}\right\}_{h \in H}\right)$, where for all $d \in D, c \in C$ and $h \in H, P_{d}$ is a preference of doctor $d, P_{c}$ is a responsive preference of couple $c$, and $P_{h}$ is a responsive preference of hospital $h$ over acceptable and feasible sets of doctors, respectively. Note that, for any hospital $h \in H, D_{h}$ is an inherent part of $P_{h}$. This means that a preference $P_{h}$ automatically specifies the acceptable set $D_{h}$ of the hospital.

A matching problem is a tuple consisting of a set of hospitals with corresponding capacities, a set of doctors with its partition into $F, M, S$, and a corresponding preference profile. 


\section{$2.3 \quad$ Stability}

There are different notions of stability based on different types of permissible blocking coalitions.

Let $\mu$ be a matching and $P$ be a preference profile. We say a hospital $h$ prefers to have a set of doctors $D^{\prime}$ (possibly empty) to a subset of doctors in $\mu(h)$ if there is $D^{\prime \prime} \subseteq \mu(h)$ with $D^{\prime} \cap D^{\prime \prime}=\emptyset$ such that $\left\{\left(\mu(h) \backslash D^{\prime \prime}\right) \cup\right.$ $\left.D^{\prime}\right\} P_{h} \mu(h)$. Similarly, we say a doctor $d$ (or a couple $c$ ) prefers a hospital $h$ to $\mu(d)$ (or a pair of hospitals $\left(h, h^{\prime}\right)$ to $\left.\mu(c)\right)$ if $h P_{d} \mu(h)\left(\right.$ or $\left.\left(h, h^{\prime}\right) P_{c} \mu(c)\right)$. Note that if a hospital prefers a set of doctors to its assignment at $\mu$, then by definition, that hospital is not matched with any of those doctors at $\mu$. Moreover, it could also be that $h$ has some unacceptable doctors $D^{\prime \prime}$ in $\mu(h)$, thus $h$ prefers $D^{\prime}=\emptyset$ to $\mu(h)$.

Similarly, if a doctor (or a couple) prefers a hospital (or a pair of hospitals) to its assignment at $\mu$, then that doctor (or at least one member of that couple) is not matched with the hospital (or the corresponding hospital) at $\mu$.

Now, we define the notion of blocking. Note that, since $\lambda$ is indifferent between all sets of doctors, and $D_{\lambda}=D$, thus $\lambda$ always prefers to have any doctor than not having that doctor. First, we introduce the notion of blocking between a hospital and a doctor in $S$.

Definition 5 Let $s \in S, h \in H \cup\{\lambda\}$ and let $\mu$ be a matching. Then $(h, s)$ blocks $\mu$ if $h$ prefers $s$ to $\mu(h)$ and $s$ prefers $h$ to $\mu(s)$.

Next, we define the notion of blocking between a pair of hospitals and a couple.

Definition 6 Let $\mu$ be a matching and let $c=\{f, m\} \in C$ and $\left(h_{f}, h_{m}\right) \in$ $(H \cup\{\lambda\})^{2}$. Then, $\left(\left(h_{f}, h_{m}\right), c\right)$ blocks $\mu$ if c prefers $\left(h_{f}, h_{m}\right)$ to $\mu(c)$ and

(i) if $h_{f} \neq h_{m}$ and $\mu(f) \neq h_{f}$, then $h_{f}$ prefers $f$ to $\mu\left(h_{f}\right)$,

(ii) if $h_{f} \neq h_{m}$ and $\mu(m) \neq h_{m}$, then $h_{m}$ prefers $m$ to $\mu\left(h_{m}\right)$, and 
(iii) if $h_{f}=h_{m}$, then $h_{f}$ prefers $\{f, m\}$ to $\mu\left(h_{f}\right)$.

It is worth mentioning that the blocking notion takes complementarity of a couple being accepted into account (by allowing the notion of a hospital being interested in a couple) but it does not take the couple into account when accepting single doctors and possibly removing members of a couple. In other words, there is an asymmetry here.

We consider this asymmetry in our model since it is not practical for big institutions like hospitals to consider the possibility of losing a member of a couple while removing the other member. This is because this possibility depends on factors like which hospital the removed member will join, whether the couple prefers to be together in that hospital, etc. Clearly, such situations can only be modeled by using a farsighted notion of blocking, which would complicate the model considerably.

Thus, by allowing the notion of a hospital being interested in a couple, the blocking definition takes complementarity of a couple being accepted into account. However, the hospital does not take this into account while accepting single doctors at the cost of removing a member of the couple from the hospital. The asymmetry arising here is the main reason, why the results obtained in this chapter are different to the results obtained by choice function approach in many-to-many matchings. ${ }^{1}$

Whenever a matching $\mu$ is blocked by $\left(\left(h_{f}, \mu(m)\right), c\right)$ for some $c=\{f, m\} \in$ $C$ and some $h_{f} \in H \cup\{\lambda\}$, for ease of presentation we say that $\mu$ is blocked by $\left(h_{f}, f\right)$. Similarly we say that $\mu$ is blocked by $\left(h_{m}, m\right)$ if $\mu$ is blocked by $\left(\left(\mu(f), h_{m}\right), c\right)$.

Our next remark follows from the responsiveness of couples' preferences and Definition 6.

REMARK 1 Let $\mu$ be a matching and $c=\{f, m\}$ be a couple. Suppose for some $x \in\{f, m\}$ and some hospital $h_{x} \in H$, we have $h_{x} P_{x} \mu(x)$ and $\left(\left(\mu\left(h_{x}\right) \backslash\right.\right.$ d) $\cup\{x\}) P_{h_{x}} \mu\left(h_{x}\right)$ for some $d \in \mu\left(h_{x}\right) \backslash\{f, m\}$, then $\left(h_{x}, x\right)$ blocks $\mu$.

\footnotetext{
${ }^{1}$ See Konishi and Ünver[11], Echenique and Oviedo[3], Hatfield and Kojima[6], for notions of stability in many-to-many matchings.
} 
A matching is stable if it cannot be blocked by any blocking pair. More formally, we get the following definition.

Definition 7 A matching $\mu$ is stable, if

(i) for all $h \in H \cup \lambda$ and $s \in S$, (h,s) does not block $\mu$,

(ii) for all $\left(h_{f}, h_{m}\right) \in(H \cup \lambda)^{2}$ and $c \in C,\left(\left(h_{f}, h_{m}\right), c\right)$ does not block $\mu$, and

(iii) for all $h \in H,(h, \emptyset)$ does not block $\mu(h)$, i.e., $h$ does not prefer $\emptyset$ to $\mu(h)$.

Now, we define the concept of individual rationality.

Definition 8 A matching $\mu$ is individually rational if

(i) for all $s \in S, \mu(s) R_{s} \lambda$,

(ii) for all $c \in C, \mu(c) R_{c}(\lambda, \lambda)$, and

(iii) for all $h \in H$ and all $d \in \mu(h), d \in D_{h}$.

The next remark follows from the definition of stability.

REMARK 2 Every stable matching is individually rational.

\subsection{Algorithm}

In this section we present a well-known algorithm called doctor proposing deferred acceptance algorithm (DPDA). This algorithm was introduced by Gale and Shapley[4]. ${ }^{2}$ Our proofs for the existence of stable matchings use a modification of DPDA. In what follows, we give a very short description of this algorithm. Take a profile $P$. Then, the DPDA algorithm at $P$ goes as follows.

\footnotetext{
${ }^{2}$ See Knuth[10], Gusfield and Irving[5], Roth and Sotomayer[14], Aldershof and Carducci[1] for additional results on stable matching problem in two sided matching.
} 
DPDA: In step 1 of the algorithm, all doctors simultaneously propose to their most preferred hospitals. Each hospital $h \in H$ provisionally accepts the most preferred doctors according to $P_{h}$. If a hospital receives more than $\kappa_{h}$ proposals, then it rejects all the doctors which do not belong to its $\kappa_{h}$ most preferred doctors. In any step $k$, the unmatched doctors propose to their most preferred hospital from the remaining set of hospitals who have not rejected them in any of the earlier steps. In any step of DPDA, since any hospital $h \in H$ accepts the most preferred collection of doctors according to $P_{h}$, it may reject some doctors that it had provisionally accepted earlier. Hospitals whose provisional list of accepted doctors is less than their maximum capacity can still add to their accepted list if they receive fresh proposals. Thus the algorithm terminates when each doctor is matched with some hospital or has been rejected by all acceptable hospitals.

REMARK 3 Note that in DPDA, each individual doctor proposes according to his/her individual preference. Thus, couples' preferences do not play any role in this algorithm.

It is well-known that the outcome of DPDA is optimal for doctors. That is, some doctor is worse off at every other stable matching. Moreover, by responsiveness and the structure of DPDA, it follows that the outcome of DPDA is individually rational.

The following remark follows directly from the definition of DPDA.

REMARK 4 Let $\mu$ be the outcome of DPDA. Let $d \notin \mu(h)$ for some $d \in D$ and $h \in H$. Then, $h P_{d} \mu(d)$ implies $d^{\prime} P_{h} d$ for all $d^{\prime} \in \mu(h)$.

In the following lemmas, we show that the outcome of DPDA cannot be blocked by a hospital and a single doctor or by a pair of different hospitals and a couple. Some of these results are well known outcomes of DPDA, but we prove them nevertheless for the sake of completeness.

LEMMA 1 The outcome of DPDA cannot be blocked by a pair $(h, s)$ for any $h \in H$ and any $s \in S$. 
Proof: Let $\mu$ be the outcome of DPDA. Assume for contradiction that $(h, s)$ blocks $\mu$ for some $h \in H$ and some $s \in S$. Since the outcome of DPDA is individually rational, $s R_{h} \lambda$. Since $h P_{s} \mu(s)$, by the definition of DPDA and Remark 4, either $s$ has not proposed to $h$ during the DPDA or all the doctors in $\mu(h)$ are preferred to $s$ according to $P_{h}$. If $s$ has not proposed to $h$ during DPDA, then we have $\mu(s) P_{s} h$, a contradiction to the fact that $(h, s)$ blocks $\mu$. So, suppose $d P_{h} s$ for all $d \in \mu(h)$. Then, by responsiveness of hospitals' preferences, we have $\mu(h) P_{h}((\mu(h) \backslash\{d\}) \cup s)$ for all $d \in \mu(h)$, and consequently, hospital $h$ will not block with $s$. This completes the proof of the lemma.

LEMMA 2 The outcome of DPDA cannot be blocked by $\left(\left(h_{1}, h_{2}\right), c\right)$ for any $h_{1}, h_{2} \in H$ such that $h_{1} \neq h_{2}$ and for any $c \in C$.

Proof: Let $\mu$ be the outcome of DPDA. Assume for contradiction that $\mu$ is blocked by $\left(\left(h_{1}, h_{2}\right), c\right)$. Let $\mu(f)=h_{f}$ and $\mu(m)=h_{m}$. By the definition of a block, $\left(h_{1}, h_{2}\right) P_{c}\left(h_{f}, h_{m}\right)$.

Suppose $h_{f} R_{f} h_{1}$ and $h_{m} R_{m} h_{2}$. Since $\left(h_{1}, h_{2}\right) \neq\left(h_{f}, h_{m}\right)$, this means $\left(h_{f}, h_{m}\right) P_{c}\left(h_{1}, h_{2}\right)$, a contradiction. Now, suppose $h_{1} P_{f} h_{f}$ or $h_{2} P_{m} h_{m}$. Without loss of generality, assume $h_{2} P_{m} h_{m}$. Since the outcome of DPDA is individually rational, $h_{2} \neq \lambda$. Because $h_{2} P_{m} h_{m}$, by Remark $4, m$ proposed to $h_{2}$ at some step of DPDA and got rejected. Since $h_{1} \neq h_{2}$, by Lemma 1 , we have $\mu\left(h_{2}\right) P_{h_{2}}\left(\left(\mu\left(h_{2}\right) \backslash\{d\}\right) \cup m\right)$ for all $d \in \mu\left(h_{2}\right)$. However, this contradicts the definition of a block.

In what follows, we give a lemma which shows that the outcome of DPDA cannot be blocked by a pair of dummy hospitals and a couple.

LEMMA 3 The outcome of DPDA cannot be blocked by $((\lambda, \lambda), c)$ for any $c \in C$.

Proof: Let $\mu$ be the outcome of DPDA. Assume for contradiction that $\mu$ is blocked by $((\lambda, \lambda), c)$. Let $\mu(f)=h_{f}$ and $\mu(m)=h_{m}$. By the definition 
of block, $(\lambda, \lambda) P_{c}\left(h_{f}, h_{m}\right)$. By responsiveness, this means $\lambda P_{x} h_{x}$ for some $x \in\{f, m\}$. However, by the definition of DPDA, $\lambda P_{x} h_{x}$ means $x$ proposed to $\lambda$ before proposing to $h_{x}$ and got rejected. This is contradiction as, by our assumption, $\lambda$ cannot reject a doctor.

In the following lemmas, we give conditions when an outcome of DPDA cannot be blocked by a pair of same hospitals and a couple.

LEMma 4 Suppose $\mu$ is an outcome of DPDA. Then for any $h \in H$ and any $c=\{f, m\} \in C$,

(i) $f P_{h} m$ and $h P_{f} \mu(f)$ implies $((h, h), c)$ cannot block $\mu$, and

(ii) $m P_{h} f$ and $h P_{m} \mu(m)$ implies $((h, h), c)$ cannot block $\mu$.

Proof: Assume without loss of generality that $f P_{h} m$ and $h P_{f} \mu(f)$. By the definition of DPDA, $f$ proposed to $h$ and got rejected. By Remark $4, d P_{h} f$ for all $d \in \mu(h)$. Because $f P_{h} m,\left\{d, d^{\prime}\right\} P_{h}\{f, m\}$ for all $d, d^{\prime} \in \mu(h)$. This means $h$ cannot block $\mu$ with $c$. This completes the proof.

LEMma 5 Suppose $\mu$ is an outcome of DPDA. Then for any $h \in H$ and any $c=\{f, m\} \in C,((h, h), c)$ cannot block $\mu$ if $h=\mu(x)$ for some $x \in\{f, m\}$

Proof: Without loss of generality, let $h=\mu(f)$. Let $((h, h), c)$ block $\mu$.

By the definition of a block, $(h, h) P_{c}(\mu(f), \mu(m))$. Thus, by responsiveness of couples' preferences, $h P_{m}^{0} h_{m}$. By the definition of DPDA, this means $m$ proposed to $h$ and got rejected. Since $f \in \mu(h)$, by Lemma 1 and the definition of block, we have $\mu(h) P_{h}((\mu(h) \backslash\{d\}) \cup m)$ for all $d \in \mu(h)$. This means $h$ will not block $\mu$ with $c$, which is a contradiction.

REMARK 5 If a doctor $d \in D$ and hospital $h \in H$ are each other's top ranked alternative, then trivially for a stable match, they must be matched to each other. 


\section{Stable matching is not guaranteed at arbi- trary responsive profiles}

In this section, we show by means of two examples that existence of a stable matching is not guaranteed at arbitrary responsive preference profiles. The two examples are slightly different to suit the subsequent sections.

ExAmple 1 Let $H=\left\{h_{1}, h_{2}, h_{3}\right\}, \kappa_{h}=2$ for all $h \in\left\{h_{1}, h_{2}, h_{3}\right\}$, and $D=\left\{d_{1}, d_{2}, d_{3}, d_{4}, f, m\right\}$ where $c=\{f, m\}$ is a couple.

Suppose $r_{1}\left(P_{d_{1}}\right)=h_{2}$ and $r_{2}\left(P_{d_{1}}\right)=r_{1}\left(P_{d_{2}}\right)=h_{1}$. Further, $r_{1}\left(P_{d_{3}}\right)=h_{2}$ and $r_{1}\left(P_{d_{4}}\right)=h_{3}$. For the couple, suppose $h_{2} P_{f} h_{1}, h_{2} P_{f} h_{3}$ and $h_{1} P_{m} h_{3}$ but $\left(h_{1}, h_{1}\right) P_{c}\left(h_{2}, h_{3}\right)$. Finally $h P_{x} \lambda$ for all $x \in\{f, m\}$ and all $h \in H$.

The above mentioned preferences along with the preferences of all the hospitals over individuals are given in Table 1. The preference of all hospitals over pairs of doctors can be any responsive preference over pairs of doctors. However $\{f, m\} P_{h_{1}}\left\{d_{1}, d_{2}\right\}$.

The couple's preference over pairs of hospitals, where one member is matched and the other one is unmatched are assumed to be responsive and ranked below the pairs of hospitals.

\begin{tabular}{cccccccccc}
\hline$P_{d_{1}}$ & $P_{d_{2}}$ & $P_{d_{3}}$ & $P_{d_{4}}$ & $P_{f}$ & $P_{m}$ & $P_{c}$ & $\bar{P}_{h_{1}}$ & $\bar{P}_{h_{2}}$ & $\bar{P}_{h_{3}}$ \\
\hline$h_{2}$ & $h_{1}$ & $h_{2}$ & $h_{3}$ & $\vdots$ & $\vdots$ & $\vdots$ & $d_{3}$ & $d_{3}$ & $d_{4}$ \\
$h_{1}$ & $\vdots$ & $\vdots$ & $\vdots$ & $h_{2}$ & $h_{1}$ & $\left(h_{1}, h_{1}\right)$ & $d_{4}$ & $d_{4}$ & $d_{3}$ \\
$\vdots$ & & & & $\vdots$ & $\vdots$ & $\vdots$ & $f$ & $f$ & $d_{1}$ \\
& & & & $h_{1}$ & $h_{3}$ & $\left(h_{2}, h_{3}\right)$ & $d_{1}$ & $d_{1}$ & $f$ \\
& & & & $\vdots$ & $\vdots$ & $\vdots$ & $d_{2}$ & $m$ & $m$ \\
& & & & & & & $m$ & $d_{2}$ & $d_{2}$ \\
\hline
\end{tabular}

Table 1

The couple and $h_{1}$ have responsive preferences. In what follows, we argue that there is no stable matching for the preference profile given in Table 1. 
Assume for contradiction, that there exists a stable matching $\mu$ for the given preference profile. By Remark 5, it must be that $\mu\left(d_{3}\right)=h_{2}$ and $\mu\left(d_{4}\right)=h_{3}$, as the doctors and hospitals are each other's top ranked alternative. Thus, there is potentially only one vacancy to be filled in $h_{2}$ and $h_{3}$ respectively.

Particularly, it is not possible for the couple to be matched to $\left(h_{2}, h_{2}\right)$ and $\left(h_{3}, h_{3}\right)$ for a stable matching. Also, since the couple prefers to be matched with any two hospitals than having at least one member of the couple unmatched and we have sufficiently many number of vacancies in all the three hospitals, thus, $\{\mu(f), \mu(m)\} \subseteq\left\{h_{1}, h_{2}, h_{3}\right\}$.

Now we look at the following allocations of the couple in $\mu$.

(i) Suppose $\mu(c)=\left(h_{2}, h_{1}\right)$.

Note that $f P_{h_{2}} d_{1}$. Since $d_{i} \bar{P}_{h_{1}} m$ for $i \in\{1,2\}, r_{2}\left(P_{d_{1}}\right)=r_{1}\left(P_{d_{2}}\right)=h_{1}$. So, $\mu\left(h_{2}\right)=\left\{d_{3}, f\right\}$. As $\kappa_{h_{1}}=2$, therefore $d_{i} \notin \mu\left(h_{1}\right)$ for some $i$. Thus, stability of $\mu$ implies that $\left(h_{1}, d_{i}\right)$ blocks $\mu$.

(ii) Suppose $\mu(c)=\left(h_{1}, h_{1}\right)$.

Then $\left(h_{1}, d_{2}\right)$ blocks $\mu$.

(iii) Suppose $\mu(c)=\left(h_{2}, h_{3}\right)$.

Since $\{f, m\} P_{h_{1}}\left\{d_{1}, d_{2}\right\}$ and $\left(h_{1}, h_{1}\right) P_{c}\left(h_{2}, h_{3}\right),\left(\left(h_{1}, h_{1}\right), c\right)$ blocks $\mu$.

(iv) Suppose $\mu(c)=\left(h_{1}, h_{3}\right)$.

Since $h_{2} P_{f} h_{1}$, responsiveness implies $\left(h_{2}, h_{3}\right) P_{c}\left(h_{1}, h_{3}\right)$. This together with the fact that $f \bar{P}_{h_{2}} d_{1} \bar{P}_{h_{2}} d_{2}$ implies $\left(\left(h_{2}, h_{3}\right), c\right)$ blocks $\mu$.

(v) Suppose $\mu(c)=\left(h_{3}, h_{1}\right)$.

Since $h_{2} P_{f} h_{3}$, responsiveness implies $\left(h_{2}, h_{1}\right) P_{c}\left(h_{3}, h_{1}\right)$. This together with the fact that $f \bar{P}_{h_{2}} d_{1} \bar{P}_{h_{2}} d_{2}$ implies $\left(\left(h_{3}, h_{1}\right), c\right)$ blocks $\mu$.

(vi) Suppose $\mu(c)=\left(h, h_{2}\right)$ for some $h \in\left\{h_{1}, h_{3}\right\}$.

Thus, $d_{1} \notin \mu\left(h_{2}\right)$. Since $r_{1}\left(P_{d_{1}}\right)=h_{2}$ and $d_{1} P_{h_{2}} m$, stability of $\mu$ implies that $\left(h_{2}, d_{1}\right)$ blocks $\mu$. 
Cases (i)-(vi) together are exhaustive. Thus, it follows that there is no stable matching for the given preference profile in Table 1.

Note that, the arguments of this example can also be used to give an example with no stable matching when we consider $h_{2}$ and $h_{3}$ as the same hospital. Thus, we just consider two vacancies for both the hospitals while excluding $d_{3}$ and $d_{4}$ from the set of doctors. It follows from the above argument that there does not exist a stable matching when $\left(h_{1}, h_{1}\right) P_{c}\left(h_{2}, h_{2}\right)$ with $h_{2} P_{f} h_{1}, h_{1} P_{m} h_{2}$ and the preferences of hospitals over $\left\{f, m, d_{1}, d_{2}\right\}$ as given by $\bar{P}_{h_{1}}$ and $\bar{P}_{h_{2}}$.

EXAMPLe 2 Let $H=\left\{h_{1}\right\}, \kappa_{h_{1}}=2$ and $D=\left\{d_{1}, d_{2}, f, m\right\}$ where $c=\{f, m\}$ is a couple.

The preference of the hospital over individuals and pairs of individuals, preference of individual doctors and the couple preferences are given in Table 2 .

The preference of $h_{1}$ over the pairs of doctors where at least one position is vacant is assumed to ranked below the shown pairs.

\begin{tabular}{ccccccc}
\hline $\bar{P}_{h_{1}}$ & $P_{d_{1}}$ & $P_{d_{2}}$ & $P_{f}$ & $P_{m}$ & $P_{c}$ & $P_{h_{1}}$ \\
\hline$f$ & $h_{1}$ & $h_{1}$ & $\lambda$ & $h_{1}$ & $\left(\lambda, h_{1}\right)$ & $\left\{f, d_{1}\right\}$ \\
$d_{1}$ & $\lambda$ & $\lambda$ & $h_{1}$ & $\lambda$ & $\left(h_{1}, h_{1}\right)$ & $\left\{f, d_{2}\right\}$ \\
$d_{2}$ & & & & & $(\lambda, \lambda)$ & $\{f, m\}$ \\
$m$ & & & & & $\left(h_{1}, \lambda\right)$ & $\left\{d_{1}, d_{2}\right\}$ \\
& & & & & & $\left\{d_{1}, m\right\}$ \\
& & & & & & $\left\{d_{2}, m\right\}$ \\
\hline
\end{tabular}

Table 2

Note that the couple and $h_{1}$ have responsive preferences In what follows, we argue that there is no stable matching for the preference profile given in Table 2. 
Assume for contradiction, that there exists a stable matching $\mu$ for the given preference profile. We look at the following allocations of the couple in $\mu$.

(i) Suppose $\mu(c)=\left(\lambda, h_{1}\right)$.

Since $d_{i} \bar{P}_{h_{1}} m, r_{1}\left(d_{i}\right)=h_{1}$ for $i \in\{1,2\}$, and $\kappa_{h_{1}}=2$, therefore $d_{i} \notin$ $\mu\left(h_{1}\right)$ for some $i$. Thus, stability of $\mu$ implies that $\left(h_{1}, d_{i}\right)$ blocks $\mu$.

(ii) Suppose $\mu(c)=(\lambda, \lambda)$.

Since $\{f, m\} P_{h_{1}}\left\{d_{1}, d_{2}\right\}$ and $\left(h_{1}, h_{1}\right) P_{c}(\lambda, \lambda),\left(\left(h_{1}, h_{1}\right), c\right)$ blocks $\mu$.

(iii) Suppose $\mu(c)=\left(h_{1}, h\right)$ for some $h \in\left\{h_{1}, \lambda\right\}$.

Since $r_{1}\left(P_{f}\right)=\lambda$, it follows by responsiveness that $((\lambda, h), c)$ blocks $\mu$.

Since cases (i)-(iii) are exhaustive, it follows that there is no stable matching for the given preference profile in Table 2 .

\section{Conditions on couples' preferences for stabil- ity}

In view of Example 1, we look for necessary and sufficient conditions on couples' preferences that guarantee the existence of a stable matching for every profile.

Let $P_{C}^{0}=\left(\left\{P_{c}^{0}\right\}_{c \in C}\right)$ be a given profile of preferences of the couples. Thus, for any $c=\{f, m\} \in C$, and a given couple preference $P_{c}^{0}, P_{f}^{0}$ and $P_{m}^{0}$ denote the individual preferences of $f$ and $m$ respectively. Since preferences of couples are responsive, a couple preference $P_{c}^{0}$ uniquely determines the individual preferences $P_{f}^{0}$ and $P_{m}^{0}$ of the members of the couple. In what follows, we present a condition on $P_{C}^{0}$ called extreme-altruism.

Definition 9 A profile of couple preferences $P_{C}^{0}$ is said to satisfy extremealtruism if for all $c=\{f, m\} \in C$, all $h \in H$ and all $h^{\prime}, h^{\prime \prime} \in H \cup\{\lambda\}$ :

(i) $h^{\prime} P_{f}^{0} h, h^{\prime} R_{f}^{0} h^{\prime \prime}, h^{\prime \prime} R_{m}^{0} \lambda$ and $\kappa_{h} \leq|D|-2$ imply $\left(h^{\prime}, h^{\prime \prime}\right) P_{c}^{0}(h, h)$, and 
(ii) $h^{\prime} P_{m}^{0} h, h^{\prime} R_{m}^{0} h^{\prime \prime}, h^{\prime \prime} R_{f}^{0} \lambda$ and $\kappa_{h} \leq|D|-2$ imply $\left(h^{\prime \prime}, h^{\prime}\right) P_{c}^{0}(h, h)$.

For illustration of extreme-altruism, consider a couple $c=\{f, m\}$ and hospitals $h \in H$ and $h^{\prime}, h^{\prime \prime} \in H \cup\{\lambda\}$. Suppose $f$ strictly prefers $h^{\prime}$ to $h$ and weakly prefers $h^{\prime}$ to $h^{\prime \prime}$, and $m$ weakly prefers $h^{\prime \prime}$ to $\lambda$. Then extreme-altruism says that, if $h$ does not have sufficiently large capacity, then $c$ ranks $\left(h^{\prime}, h^{\prime \prime}\right)$ above $(h, h)$. Note here, that if $h$ has a sufficiently large capacity, then we would not require any restriction on couples' preferences, simply because the hospital $h$ will have enough vacancies to accommodate at least one member of the couple, thereby removing the possibility of the couple to block with the hospital.

Out of two allocations of a couple, one where both the members are allocated at the same hospital and the other where one member moves to a better hospital (according to his/her individual preference), the couple prefers the latter to the former. For instance, if the hospital $h^{\prime}$ gives a substantially better salary compared to $h$. Then the couple would rather prefer on member to be at $h^{\prime}$ than both the members of the couple to be at $h$.

Our next lemma shows that extreme-altruism and responsiveness together imply that if two hospitals are acceptable for both members of a couple, then the ranking of those two hospitals by each member of the couple is always the same. That is, if $h_{1}$ and $h_{2}$ are both acceptable by each member of a couple $\{f, m\}$, then $f$ and $m$ will have same ranking over $h_{1}$ and $h_{2}$.

LEMMA 6 Let $P_{C}^{0}$ be a profile of couple preferences satisfying extreme-altruism. Then, for any $c=\{f, m\}$ and any $h_{1}, h_{2} \in H$ such that $h P_{x}^{0} \lambda$ for all $x \in\{f, m\}$ and all $h \in\left\{h_{1}, h_{2}\right\}$, we have $h_{1} P_{f}^{0} h_{2}$ if and only if $h_{1} P_{m}^{0} h_{2}$.

Proof: Let $P_{C}^{0}$ be a profile of couple preferences satisfying extreme-altruism. Consider a couple $c=\{f, m\}$ with preference $P_{c}^{0}$ and consider two hospitals $h_{1}, h_{2} \in H$. Assume for contradiction $h_{1} P_{f}^{0} h_{2} P_{f}^{0} \lambda$ and $h_{2} P_{m}^{0} h_{1} P_{m}^{0} \lambda$. By responsiveness and Condition (i) of the definition of extreme-altruism, we have $\left(h_{1}, \lambda\right) P_{c}^{0}\left(h_{2}, h_{2}\right) P_{c}^{0}\left(\lambda, h_{2}\right)$. Again, responsiveness and Condition (ii) 
of extreme-altruism implies $\left(\lambda, h_{2}\right) P_{c}^{0}\left(h_{1}, h_{1}\right) P_{c}^{0}\left(h_{1}, \lambda\right)$. However, this is a contradiction.

Our next corollary follows directly from Lemma 6. It says the following. Suppose $P_{C}^{0}$ satisfies the extreme-altruism. Consider a couple. Suppose that every hospital is acceptable for each member of the couple. Then, the members of the couple have the same individual preference over $H$.

COROLlaRY 1 Let $P_{C}^{0}$ be a profile of couple preferences satisfying extremealtruism. Let $c=\{f, m\} \in C$ be a couple such that $h P_{f}^{0} \lambda$ and $h P_{m}^{0} \lambda$ for all $h \in H$. Then $P_{m}^{0}=P_{f}^{0}$.

For a profile of preferences $P_{C}^{0}$ of the couples, an extension of $P_{C}^{0}$ is defined as a preference profile $P=\left(\left\{P_{d}\right\}_{d \in D},\left\{P_{c}\right\}_{c \in C},\left\{P_{h}\right\}_{h \in H}\right)$ such that $P_{c}=P_{c}^{0}$ for all $c \in C$.

The following theorem says that extreme-altruism of $P_{C}^{0}$ is necessary and sufficient for the existence of a stable matching for every extension of $P_{C}^{0}$.

THEOREM 1 (i) If $P_{C}^{0}$ satisfies extreme-altruism, then a stable matching exists for any extension of $P_{C}^{0}$.

(ii) If $P_{C}^{0}$ does not satisfy extreme-altruism for all $h \in H$, then there exists an extension of $P_{C}^{0}$ with no stable matching.

Proof: [Part (i)] Consider a preference profile $P$ that is an arbitrary extension of $P_{C}^{0}$ where $P_{C}^{0}$ satisfies extreme-altruism. We show that the DPDA where each member of each couple proposes according to his/her individual preference gives a stable matching for $P$.

Let $\mu$ be the outcome. Suppose $\mu$ is not stable at $P$. Since DPDA is individually rational, Lemma 1, Lemma 2 and Lemma 3 imply that $\mu$ is blocked by $((h, h), c)$ for some $h \in H$ and some $c=\{f, m\} \in C$. Let $\mu(f)=h_{f}$ and $\mu(m)=h_{m}$. Thus $(h, h) P_{c}^{0}\left(h_{f}, h_{m}\right)$.

Assume without loss of generality that $f P_{h} m$. By Lemma 4 and Lemma 5 , we know that $h_{f} P_{f}^{0} h$. Thus, by responsiveness, we must have $h P_{m}^{0} h_{m}$. 
Suppose $\kappa_{h}>|D|-2$. Then $\{f, m\} \notin \mu(h)$ implies $|\mu(h)| \leq|D|-2<\kappa_{h}$. But since $h P_{m}^{0} h_{m}, m$ proposed to $h$ at an earlier step of DPDA and got rejected even when $h$ had a vacancy. Thus $m \notin D_{h}$ which implies that $((h, h), c)$ cannot block $\mu$. Thus $\kappa_{h} \leq|D|-2$.

By the definition of DPDA, we have $h_{f} R_{f}^{0} \lambda$ and $h_{m} R_{m}^{0} \lambda$. Also, we know that $x$ is weakly preferred to itself. This together with extreme-altruism implies $\left(h_{f}, \lambda\right) P_{c}^{0}(h, h) P_{c}^{0}\left(h_{f}, h_{m}\right)$.

This contradicts the fact that $h_{m} R_{m}^{0} \lambda$. Thus $\left(h_{f}, h_{m}\right) R_{c}^{0}\left(h_{f}, \lambda\right) P_{c}^{0}(h, h)$ which contradicts that $((h, h), c)$ blocks $\mu$. This completes the proof of part (i) of Theorem 1.

[Part (ii)] Suppose $P_{C}^{0}$ does not satisfy extreme-altruism. We show that there exists an extension of $P_{C}^{0}$ with no stable matching.

Since $P_{C}^{0}$ does not satisfy extreme-altruism, there is a couple $c=\{f, m\}$, a hospital $h$ such that $\kappa_{h} \leq|D|-2$ and hospitals $h_{1}, h_{2} \in H \cup\{\lambda\} \backslash\{h\}$ such that:

(i) either, $h_{1} P_{f}^{0} h, h_{1} R_{f}^{0} h_{2}$ and $h_{2} R_{m}^{0} \lambda$, but $(h, h) P_{c}^{0}\left(h_{1}, h_{2}\right)$,

(ii) or, $h_{1} P_{m}^{0} h, h_{1} R_{m}^{0} h_{2}$ and $h_{2} R_{f}^{0} \lambda$, but $(h, h) P_{c}^{0}\left(h_{2}, h_{1}\right)$.

Assume without loss of generality that (i) holds.

By responsiveness, $h_{1} P_{f}^{0} h$ and $(h, h) P_{c}^{0}\left(h_{1}, h_{2}\right)$, implies $h P_{m}^{0} h_{2}$. Consider a preference profile $P$ such that

(i) for all $h^{\prime} \in H \backslash\left\{h, h_{1}, h_{2}\right\}$, either $\mid\left\{d: d P_{h} c\right.$ and $\left.r_{1}\left(P_{d}\right)=h\right\} \mid=\kappa_{h^{\prime}}$ or $f, m \notin D_{h^{\prime}}$,

(ii) there are doctors $d_{1}, d_{2} \in D \backslash\{f, m\}$ such that $f P_{h} d_{1} P_{h} d_{2} P_{h} m$ and $\{f, m\} P_{h}\left\{d_{1}, d_{2}\right\}$,

(iii) $\mid\left\{d: d P_{h} f\right.$ and $\left.r_{1}\left(P_{d}\right)=h\right\} \mid=\kappa_{h}-2$. This is possible since $\kappa_{h} \leq|D|-2$ implies $\kappa_{h}-2 \leq|D|-4$,

(iv) either $f P_{h_{1}} d_{1} P_{h_{1}} m$ and $\mid\left\{d: d P_{h_{1}} f\right.$ and $\left.r_{1}\left(P_{d}\right)=h_{1}\right\} \mid=\kappa_{h_{1}}-1$, or $r_{1}\left(P_{h_{1}}\right)=f, r_{2}\left(P_{h_{1}}\right)=d_{1}$ and $m \notin D_{h_{1}}$, 
(v) either $\mid\left\{d: d P_{h_{2}} c\right.$ and $\left.r_{1}\left(P_{d}\right)=h_{2}\right\} \mid=\kappa_{h_{2}}-1$ or $f \notin D_{h_{2}}$,

(vi) $r_{1}\left(P_{d_{1}}\right)=h_{1}$ and $r_{2}\left(P_{d_{1}}\right)=r_{1}\left(P_{d_{2}}\right)=h$.

But it trivially follows from this preference profile that for a stable matching $\mu, h^{\prime} \notin\{\mu(f), \mu(m)\}$ for $h^{\prime} \notin\left\{h, h_{1}, h_{2}\right\}$. Also, it is not possible that $\mu(c)=\left(h_{i}, h_{i}\right)$ for $i \in\{1,2\}$. Thus, by our construction, a stable matching exists for this matching problem if and only if there is a stable matching for Example 1. However, since there does not exist a stable matching for Example 1, thus, we do not have a stable matching for $P$. This completes the proof of part (ii) of Theorem 1.

From the above example, it is clear that even if all hospitals view all the doctors as acceptable, violation of extreme-altruism can lead to a preference profile with no stable matching, if we have enough doctors to fulfil the capacity constraints as given by points (i),(ii), (iv) and (v). Thus we get the following corollary.

Corollary 2 Suppose $D_{h}=D$ for all $h \in H$. Moreover, $\sum_{h \in H} \kappa_{h}=|D|$, then:

(i) If $P_{C}^{0}$ satisfies extreme-altruism, then a stable matching exists for any extension of $P_{C}^{0}$.

(ii) If $P_{C}^{0}$ does not satisfy extreme-altruism for all $h \in H$, then there exists an extension of $P_{C}^{0}$ with no stable matching.

\section{Conditions on hospitals' preferences for sta- bility}

In Section 4, we have discussed a necessary and sufficient condition on couples' preferences that guarantees the existence of a stable matching for every collection of preferences of the hospitals. In this section, we look at the other 
side of the problem, that is, we look for necessary and sufficient condition on hospitals' preferences so that a stable matching exists for every collection of preferences of the doctors (both individuals and couples).

Let $P_{H}^{0}=\left(\left\{P_{h}^{0}\right\}_{h \in H}\right)$ be a given profile of preferences of the hospitals. In what follows, we introduce the aversion to couple diversity property.

Definition 10 A profile of hospital preferences $P_{H}^{0}$ is said to have aversion to couple diversity if for all $h \in H$, all $c=\{f, m\}$ and all $d_{1}, d_{2} \in D$ with $f, m \in D_{h}$ such that either (i) $f P_{h}^{0} d_{1} P_{h}^{0} d_{2} P_{h}^{0} m$ and $\left|\left\{d: d P_{h}^{0} m\right\}\right|>\kappa_{h}$, or (ii) $m P_{h}^{0} d_{1} P_{h}^{0} d_{2} P_{h}^{0} f$ and $\left|\left\{d: d P_{h}^{0} f\right\}\right|>\kappa_{h}$, we have $\left\{d_{1}, d_{2}\right\} P_{h}^{0}\{f, m\}$.

Consider a hospital $h$ with a preference $P_{h}$ over acceptable and feasible sets of doctors. Take a couple $c=\{f, m\}$ such that both $f$ and $m$ are acceptable for $h$ but at least one of them is not amongst the top- $\kappa_{h}$ doctors according to the restriction of $P_{h}$ over individual doctors. Suppose that there are two doctors $d_{1}, d_{2}$ who are ranked in-between $f$ and $m$ according to $P_{h}$. Then, aversion to couple diversity says that the set $\left\{d_{1}, d_{2}\right\}$ must be preferred to the couple $c$ according to $P_{h}$. Note here that if both the members of the couple are in the top- $\kappa_{h}$ doctors according to the restriction of $P_{h}$ over individual doctors, then we do not need this condition as for a stable matching, the couple will always be a part of $h$.

So, in other words, whenever a hospital compares a couple and another pair of doctors over which responsiveness does not induce the comparison, the hospital prefers the couple only if at most one doctor from the other pair ranks in-between the members of the couple. Thus, a hospital has aversion to couple diversity if it does not like to employ a couple whose members have relatively more dissimilar ranking in its preference.

It is important to note here that the diversity aversion just applies to couples and not single doctors as two single doctors can not apply to a hospital together and block a matching. On the other hand, we can encounter a situation where a couple applies to a hospital such that a member of a couple is individually worse off but the couple is better off as a whole. 
For a profile of preferences $P_{H}^{0}$ of the hospitals, an extension of $P_{H}^{0}$ is defined as a preference profile $P=\left(\left\{P_{d}\right\}_{d \in D},\left\{P_{c}\right\}_{c \in C},\left\{P_{h}\right\}_{h \in H}\right)$ such that $P_{h}=P_{h}^{0}$ for all $h \in H$.

Our next theorem says that the aversion to couple diversity of $P_{H}^{0}$ is necessary and sufficient for the existence of a stable matching for every extension of $P_{H}^{0}$.

THEOREM 2 (i) If $P_{H}^{0}$ satisfies aversion to couple diversity property, then a stable matching exists at every extension of $P_{H}^{0}$.

(ii) If $P_{H}^{0}$ does not satisfy aversion to couple diversity property, then there exists an extension of $P_{H}^{0}$ with no stable matching.

Proof: [Part (i)] Consider a preference profile $P$ that is an arbitrary extension of $P_{H}^{0}$ where $P_{H}^{0}$ satisfies aversion to couple diversity. We show that the DPDA where each member of each couple proposes according to his/her individual preference gives a stable matching for $P$.

Let $\mu$ be the outcome. Suppose $\mu$ is not stable at $P$. Since DPDA is individually rational, Lemma 1 , Lemma 2 and Lemma 3 imply that $\mu$ is blocked by $((h, h), c)$ for some $h \in H$ and some $c=\{f, m\} \in C$. Let $\mu(f)=h_{f}$ and $\mu(m)=h_{m}$. Thus $(h, h) P_{c}^{0}\left(h_{f}, h_{m}\right)$.

Assume without loss of generality that $f P_{h} m$. If $m \notin D_{h}$, then $((h, h), c)$ cannot block $\mu$ as it violates individual rationality. Thus $m P_{h}^{0} \lambda$. By Lemma 4 and Lemma 5, we know that $h_{f} P_{f}^{0} h$. Thus, responsiveness implies $h P_{m} h_{m}$.

It follows that, before applying to $h_{m}, m$ applied to $h$ and got rejected. Therefore, $\mid\left\{d: d P_{h}^{0} m\right.$ and $\left.d \in \mu(h)\right\} \mid=\kappa_{h}$. Since $f P_{h}^{0} m$ and $f \notin \mu(h)$, we have $\left|\left\{d: d P_{h}^{0} f\right\}\right|>\kappa_{h}$. This, together with aversion to couple diversity, implies that $\left\{d, d^{\prime}\right\} P_{h}^{0}\{f, m\}$ for all $d, d^{\prime} \in \mu(h)$, which is a contradiction to the fact that $((h, h), c)$ blocks $\mu$. This completes the proof of part (i) of Theorem 2.

[Part (ii)] Suppose $P_{H}^{0}$ does not satisfy the aversion to couple diversity property. We show that there is an extension of $P_{H}^{0}$ with no stable matching. 
Since $P_{H}^{0}$ does not satisfy the aversion to couple diversity property, we have $h \in H, c=\{f, m\} \in C$ and $d_{1}, d_{2} \in D$ with $f, m \in D_{h}$ such that $\{f, m\} P_{h}^{0}\left\{d_{1}, d_{2}\right\}$ and either

(i) $f P_{h}^{0} d_{1} P_{h}^{0} d_{2} P_{h}^{0} m$ and $\left|\left\{d: d P_{h}^{0} m\right\}\right|>\kappa_{h}$, or

(ii) $m P_{h}^{0} d_{1} P_{h}^{0} d_{2} P_{h}^{0} f$ and $\left|\left\{d: d P_{h}^{0} f\right\}\right|>\kappa_{h}$.

Assume without loss of generality that (i) holds. Consider a preference profile $P$ such that

(i) $\lambda P_{f} h$,

(ii) $h P_{m} \lambda$,

(iii) $(h, h) P_{c}(\lambda, \lambda)$,

(iv) for all $h^{\prime} \in H \backslash\{h\}$ either $h P_{f} h^{\prime}$, or $\mid\left\{d: d P_{h^{\prime}}^{0} f\right.$ and $\left.r_{1}\left(P_{d}\right)=h^{\prime}\right\} \mid=\kappa_{h^{\prime}}$,

(v) for all $h^{\prime} \in H \backslash\{h\}$ either $\lambda P_{m} h^{\prime}$, or $\mid\left\{d: d P_{h^{\prime}}^{0} m\right.$ and $\left.r_{1}\left(P_{d}\right)=h^{\prime}\right\} \mid=$ $\kappa_{h^{\prime}}$,

(vi) $r_{1}\left(P_{d_{1}}\right)=r_{1}\left(P_{d_{2}}\right)=h$, and

(vii) $\mid\left\{d: d P_{h}^{0} m\right.$ and $\left.r_{1}\left(P_{d}\right)=h\right\} \mid=\kappa_{h}$. Note, that this also includes $d_{1}$ and $d_{2}$.

But it trivially follows from the given preference profile that for a stable matching $\mu$, for all $h^{\prime} \in H \backslash\{h\}, h^{\prime} \notin\{\mu(f), \mu(m)\}$. Thus, by our construction, a stable matching exists for this matching problem if and only if there is a stable matching for Example 2. However, since there does not exist a stable matching for Example 2, thus we do not have a stable matching for $P$. This completes the proof of part (ii) of Theorem 2.

From the example above, it is not clear if non-aversion to couple diversity can always lead to a preference profile with no stable matching when doctors prefer to be matched any hospital than being unemployed. We show by the means of an example that if all the doctors are averse to unemployment, then 
we can not always obtain an extension of $P_{H}^{0}$ with no stable matching, when $P_{H}^{0}$ does not satisfy aversion to couple diversity.

ExAmple 3 Consider a matching problem with $H=\left\{h_{1}, h_{2}\right\}$ and $D=$ $\left\{f, m, s_{1}, s_{2}\right\}$ such that $c=\{f, m\}$ is the only couple. Let $\kappa_{h_{1}}=\kappa_{h_{2}}=2$. Thus, $\sum_{h \in H} \kappa_{h}=|D|$. The preferences of hospitals on individual doctors is given in the table below. The doctors prefer to be matched to any hospital than being unemployed.

\begin{tabular}{cc}
\hline$P_{h_{1}}$ & $P_{h_{2}}$ \\
\hline$f$ & $s_{2}$ \\
$s_{1}$ & $m$ \\
$s_{2}$ & $f$ \\
$m$ & $s_{1}$ \\
\hline
\end{tabular}

Let $\{f, m\} P_{h_{1}}\left\{s_{1}, s_{2}\right\}$. Thus the preference $h_{1}$ does not follow aversion to couple diversity.

We show that there exists a stable matching for these preferences of hospitals for any preferences of the doctors and the couple.

Let $\mu$ be a matching for the given preferences of the hospitals such that $\mu\left(h_{1}\right)=\left\{f, s_{1}\right\}$ and $\mu\left(h_{2}\right)=\left\{s_{2}, m\right\}$. Clearly, $h_{1}$ and $h_{2}$ have their top ranked doctors. Thus, neither $h_{1}$ nor $h_{2}$ would like to block $\mu$ with any other doctor. Also, no doctor would block $\mu$ with $\lambda$ as all doctors prefer being matched to any hospital than be unemployed. Thus $\mu$ is stable for any preferences of doctors even when there is no aversion for diverse couples.

The above example leads to the following corollary.

Corollary 3 Suppose $h P_{d} \lambda$ for all $h \in H$ and all $d \in D$, then a stable matching always exists for any extension of $P_{H}^{0}$ when $P_{H}^{0}$ satisfies aversion to diverse couples. 


\section{Concluding remarks}

As we have discussed earlier, existence of a stable matching is guaranteed at a preference profile if it satisfies responsiveness as defined in Klaus and Klijn[9]. The different result in our paper stems from the fact that we allow for a setwise blocking notion, which allows for the existence of more blocking coalitions. Here, a hospital is allowed to replace two doctors by a couple, whilst Klaus and Klijn consider pairwise blocking. Thus, if a couple wishes to block with a hospital, both the members of the couple will be considered separately by the hospital instead of considering the couple as a whole. We explain this in detail in the following paragraph.

Consider Example 1 and Example 2. According to the model in Klaus and Klijn[9], given the preference $\bar{P}_{h_{1}}$, the pair $\{f, m\}$ can not block with $h_{1}$ to remove the pair of doctors $\left\{d_{1}, d_{2}\right\}$. By their blocking notion, each member of the couple can only replace a doctor who is ranked lower to that member of the couple. The fact that $d_{1}$ and $d_{2}$ are ranked in-between $f$ and $m$, prevent the couple to block with $h_{1}$.

Now, consider the matching $\mu_{1}$ for Example 1 and $\mu_{2}$ for Example 2 such that $\mu_{1}(c)=\left(h_{2}, h_{3}\right), \mu_{1}\left(d_{1}\right)=\mu_{1}\left(d_{2}\right)=h_{1}$ and $\mu_{2}(c)=(\lambda, \lambda), \mu_{2}\left(d_{1}\right)=$ $\mu_{2}\left(d_{2}\right)=h_{1}$. Note that, in our model, both these matchings are blocked by $\left(\left(h_{1}, h_{1}\right), c\right)$. However, this block is not possible according to the model in Klaus and Klijn[9]. It can be verified that $\mu_{1}$ and $\mu_{2}$ are indeed stable according to their model.

In this paper, we have shown that the existence of a stable matching is not guaranteed when couples and/or hospitals have complete and responsive preferences. We have provided (a) necessary and sufficient conditions on couples' preferences so that a stable matching exists at every extension of those preferences, and (b) necessary and sufficient conditions on hospitals' preferences so that a stable matching exists at every extension of those preferences. Additionally, we have provided algorithms that produce a stable matching whenever that exists in this framework. 


\section{References}

[1] Brian Aldershof and Olivia M. Carducci, Stable matchings with couples, Discrete Applied Mathematics 68 (1996), 203-207.

[2] Ahmet Alkan and David Gale, Stable schedule matching under revealed preferences, Journal of Economic Theory 112 (2003), 289-306.

[3] Federico Echenique and Jorge Oviedo, A theory of stability in many-tomany matching markets, Theoretical Economics 1 (2006), no. 2, 233273.

[4] David Gale and Lloyd S Shapley, College admissions and the stability of marriage, American mathematical monthly (1962), 9-15.

[5] Dan Gusfield and Robert W. Irving, The stable marriage problem: Structure and algorithms, (1989).

[6] John William Hatfield and Fuhito Kojima, Substitutes and stability for matching with contracts, Journal of Economic Theory 145 (2010), no. 5, 1704-1723.

[7] John William Hatfield and Paul R. Milgrom, Matching with contracts, American Economic Review 95.

[8] Alexander S Kelso Jr and Vincent P Crawford, Job matching, coalition formation, and gross substitutes, Econometrica: Journal of the Econometric Society (1982), 1483-1504.

[9] Bettina Klaus and Flip Klijn, Stable matchings and preferences of couples, Journal of Economic Theory 121 (2005), no. 1, 75-106.

[10] Donald E. Knuth, Marriages stables, (1976).

[11] Hideo Konishi and M. Utku Ünver, Credible group stability in manyto-many matching problems, Journal of Economic Theory 129 (2006), $57-80$. 
[12] Alvin E. Roth, The evolution of the labor market for medical interns and residents: A case study in game theory, The Journal of Political Economy 92 (1984), 991-1016.

[13] _ The college admissions problem is not equivalent to the marriage problem, Journal of Economic Theory 36 (1985), 277-288.

[14] Alvin E. Roth and Marilda A. Oliveira Sotomayor, Two-sided matching: A study in game-theoretic modeling and analysis, no. 18, Cambridge University Press, 1992. 